\title{
Non-invasive detection of a femoral-to-radial arterial pressure gradient in intensive care patients with vasoactive agents
}

Matthias Jacquet-Lagrèze ${ }^{1,2,3}$, David Claveau4, Julie Cousineaư ${ }^{5}$, Kun Peng Liu' ${ }^{6}$, Jean-Gilles Guimond ${ }^{5}$, Pierre Aslanian ${ }^{5}$, Yoan Lamarche ${ }^{7}$, Martin Albert ${ }^{7,8}$, Emmanuel Charbonney $^{5}$, Ali Hammoud ${ }^{7}$, Loay Kontar ${ }^{9}$ and André Denault ${ }^{7 *}$ (D)

\begin{abstract}
Background: In patient requiring vasopressors, the radial artery pressure may underestimate the true central aortic pressure leading to unnecessary interventions. When using a femoral and a radial arterial line, this femoral-to-radial arterial pressure gradient (FR-APG) can be detected. Our main objective was to assess the accuracy of non-invasive blood pressure (NIBP) measures; specifically, measuring the gradient between the NIBP obtained at the brachial artery and the radial artery pressure and calculating the non-invasive brachial-to-radial arterial pressure gradient (NIBR-APG) to detect an FR-APG. The secondary objective was to assess the prevalence of the FR-APG in a targeted sample of critically ill patients.
\end{abstract}

Methods: Adult patients in an intensive care unit requiring vasopressors and instrumented with a femoral and a radial artery line were selected. We recorded invasive radial and femoral arterial pressure, and brachial NIBP. Measurements were repeated each hour for $2 \mathrm{~h}$. A significant FR-APG (our reference standard) was defined by either a mean arterial pressure (MAP) difference of more than $10 \mathrm{mmHg}$ or a systolic arterial pressure (SAP) difference of more than $25 \mathrm{mmHg}$. The diagnostic accuracy of the NIBR-APG (our index test) to detect a significant FR-APG was estimated and the prevalence of an FR-APG was measured and correlated with the NIBR-APG.

Results: Eighty-one patients aged 68 [IQR 58-75] years and an SAPS2 score of 35 (SD 7) were included from which 228 measurements were obtained. A significant FR-APG occurred in 15 patients with a prevalence of $18.5 \%[95 \% \mathrm{Cl}$ 10.8-28.7\%]. Diabetes was significantly associated with a significant FR-APG. The use of a $11 \mathrm{mmHg}$ difference in MAP between the NIBP at the brachial artery and the MAP of the radial artery led to a specificity of $92 \%$ [67; 100], a sensitivity of $100 \%$ [95\%Cl 83; 100] and an AUC ROC of 0.93 [95\%Cl 0.81-0.99] to detect a significant FR-APG. SAP and MAP FR-APG correlated with SAP $\left(r^{2}=0.36 ; p<0.001\right)$ and MAP $\left(r^{2}=0.34 ; p<0.001\right)$ NIBR-APG.

Conclusion: NIBR-APG assessment can be used to detect a significant FR-APG which occur in one in every five critically ill patients requiring vasoactive agents.

*Correspondence: andre.denault@umontreal.ca

${ }^{7}$ Montreal Heart Institute, Université de Montréal, 5000 rue Belanger, Montreal, QC H1T 1C8, Canada

Full list of author information is available at the end of the article

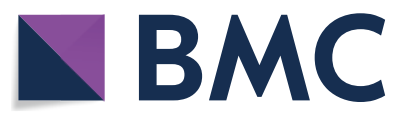

(c) The Author(s) 2021. Open Access This article is licensed under a Creative Commons Attribution 4.0 International License, which permits use, sharing, adaptation, distribution and reproduction in any medium or format, as long as you give appropriate credit to the original author(s) and the source, provide a link to the Creative Commons licence, and indicate if changes were made. The images or other third party material in this article are included in the article's Creative Commons licence, unless indicated otherwise in a credit line to the material. If material is not included in the article's Creative Commons licence and your intended use is not permitted by statutory regulation or exceeds the permitted use, you will need to obtain permission directly from the copyright holder. To view a copy of this licence, visit http://creativecommons.org/licenses/by/4.0/. The Creative Commons Public Domain Dedication waiver (http://creativeco mmons.org/publicdomain/zero/1.0/) applies to the data made available in this article, unless otherwise stated in a credit line to the data. 


\section{Key messages}

A significant radial-to-femoral pressure gradient occurs in nearly one-fifth of patients in ICU. Repeated non-invasive assessment of the radial-to-brachial pressure gradient was significantly correlated with the invasive radio-femoral arterial pressure gradient.

Keywords: Diagnostic study, Aorto-radial gradient, Femoro-radial gradient, Vasoplegia

\section{Background}

Radial arterial pressure monitoring is a common practice in intensive care units (ICU) [1]. However, the radial artery blood pressure measurement can be inaccurate. In normal patients central-to-peripheral gradient with higher pressure in peripheral artery than in the aorta is typically observed [2]. This gradient typically affects the systolic component of the arterial pressure (SAP), while the mean arterial pressure (MAP) and diastolic arterial pressures (DAP) are usually unaltered. This peripheral-to-central gradient decreases with age [3]. During cardiopulmonary bypass [4-6] or during acute circulatory failure requiring high levels of vasopressors $[7,8]$, it has been reported that a significant abnormal central to peripheral gradient could occur, with higher pressure in the aorta compared to the peripheral artery. Unfortunately, in that case, all the components of the arterial pressure can be affected, and the clinician may underestimate the central arterial pressure, if one relies only on the radial artery pressure measurement.

Risk factors in detecting this abnormal arterial peripheral-to-central gradient have been identified during cardiac surgery, but the diagnostic accuracy of such an approach is low $[9,10]$. This blood pressure (BP) gradient has significant clinical implication as it could lead the clinician to under evaluate the arterial BP leading to excessive fluid and/or vasopressor therapy. No test with enough accuracy $[11,12]$ can detect a significant central to peripheral arterial pressure gradient. Furthermore, the prevalence in the ICU of a significant peripheral to central arterial pressure gradient is rarely reported $[7,8,13$, 14]. As an alternative to radial invasive measurement, it has been reported that invasive and non-invasive blood pressure (NIBP) at brachial artery were higher $[15,16]$ and a better reflection of aortic measurement $[17,18]$. Indeed, the pressure gradient between central arterial pressure and a point of measurement in the upper limb is proportional to the distance from the aorta to the point of measurement [19].

These observations raise the question about the possibility to detect such a gradient in clinical practice, and eventually identify the population which need an invasive assessment, beyond the sole radial measurement. Several studies have shown that femoral and aortic arterial pressure were equivalents $[9,17]$, but they have not been compared to NIBP measurements. The primary objective of the present study was to assess the accuracy of non-invasive assessment of the brachial-to-radial arterial pressure gradient (NIBR-APG) to detect the femoral-toradial arterial pressure gradient (FR-APG) measured with invasive dual radial and femoral arterial BP monitoring. Secondary objectives were to correlate FR-APG and NIBR-APG and to evaluate the FR-APG prevalence in a sample of critically ill patients.

\section{Materials and methods \\ Patients and settings}

This prospective study was conducted in Montreal, Canada in three academic medical centers: over a 22-month period. The local Institutional Review Board for human subjects approved the study protocol (IRB\#: MP-332016-1964) and informed consent was obtained before inclusion. We reported our study, regarding the methodological limits and the risk of bias, according to the STARD statement $[20,21]$. Patients were eligible for inclusion if instrumented with a radial and a femoral arterial catheter and requiring a vasopressor to maintain a MAP of more than $65 \mathrm{mmHg}$. In one cardiac surgical institution, it was common practice to have both radial and femoral arterial monitoring, in the two other institutions the dual monitoring was at the discretion of the clinician in charge of the patient, mainly motivated by a suspicion of an FR-APG in patients facing high doses of vasopressors [22]. They were excluded in case of upper or lower limb amputation, arterial stenosis of the upper or lower limb, mechanical circulatory support, a moribund state, or when investigators were not available. We stopped all measurements (at T1, T2 or T3) once the vasoactive agents were weaned.

\section{Study protocol}

The experimental protocol was as follows: when the patient was stabilized, we took exactly at the same time the two values of the radial and femoral arterial pressure (Philips M1006B Invasive BP Measurement Module InteliView MX; Philips, Amsterdam, Netherlands). This was collected right after the end of the NIBP done by the attending nurse using non-invasive oscillometric arterial pressure measured with an adapted cuff size at the brachial level on both sides. All the pressure measurements were recorded in semi-recumbent position within less 
than 10 min. Invasive arterial pressure was measured with an arterial catheter connected to a pressure transducer placed at the level of the phlebostatic axis (right atrium level: 4th intercostal along the mid axillary line), zero was set at the atmospheric pressure, a fast flush test was carried out before each measurement to check the harmonic characteristic of the system. Over-damping (less than 1 oscillation after the flush test) or underdamping (more than 2 oscillations after the flush test) were identified and corrected before measurement. The invasive and NIBP data were collected simultaneously three times with $1 \mathrm{~h}$ between each measurement at T1, T2, and T3. In most cases, T1 occurs in the first hours of the ICU stay. Notification of the invasive and non-invasive arterial pressure was not recorded blindly. At each timepoints, the heart rate, the ventilatory support variables, the doses of vasopressor and inotropes were recorded. The age, height, weight, reason for admission to the ICU and associated medical conditions were also collected. Lactate, creatinine, central venous oxygen saturation $\left(\mathrm{ScVO}_{2}\right)$, cerebral near-infrared spectroscopy (NIRS), fluid balance at the time of the measurement if available and central body temperature values were noted. The Sequential Organ Failure Assessment Score [23] at inclusion and new Simplified Acute Physiology Score (SAPS2) [24] were collected. The FR-APG was defined as the difference between the invasive femoral and radial arterial pressure. The NIBR-APG was defined as the difference between the average of the NIBP recorded on the right and left upper limb and the radial arterial pressure. All the gradients were calculated based on both on the SAP and MAP.

\section{Endpoints and definitions}

The primary objective was to evaluate the diagnostic accuracy of the NIBR-APG (our index test) to detect the FR-APG (our reference standard) in patients in ICU. Secondary objectives were to evaluate the correlation between the FR-APG and the NIBR-APG and to determine the prevalence of the FR-APG in our sample of patients. A significant BP gradient was defined as either an FR-APG of more than $25 \mathrm{mmHg}$ using SAP or an FRAPG of more than $10 \mathrm{mmHg}$ using MAP $[9,10]$.

\section{Statistical analysis}

Sample size calculation was based on our primary objective. Using Obuchowsky's method [25], 81 patients were needed to detect an area under curve (AUC) of the receiver operating characteristic (ROC) curve of 0.75 with a power of 0.8 and an alpha risk of 0.05 . The ratio between patients with a significant FR-APG and patients without an FR-APG was hypothesized to be 0.15 . The Shapiro-Wilk test determined the normal distribution of the data. For continuous data, the two-tailed Student $t$ test or a Mann-Whitney $U$ test to compare patients with and without a significant RCG. In the case of categorical data, we used a Chi ${ }^{2}$ test. Pearson's correlation coefficient was used to test linear correlations. We built ROC curves and expressed AUC with 95\% confidence interval (CI) calculated with a bootstrap method using 2000 repetitions. Sensitivity, specificity, positive and negative predictive values were expressed with 95\%CI. We used the Free Software Foundation's CRAN R to compute the statistical analysis. All tests were two-sided and a $p$-value $<0.05$ was considered statistically significant.

\section{Results}

The flow chart of the study is reported in Fig. 1. A total of eighty-one patients were analyzed in which 228 measurements were obtained. The main demographic and clinical characteristics of all patients and those without and with a significant FR-APG are detailed in Table 1. Sixtyeight (84\%) patients had benefited from cardiac surgery and thirteen were non-surgical cardiac patient mainly septic shock. We found 11 patients after cardiac surgery and 4 among non-cardiac surgery patients with a significant FR-APG. Patient with a significant FR-APG had significantly more diabetes ( $53 \%$ versus $23 \%, p=0.044$ ), higher lactate $(2.8[1.6,5.2] \mathrm{mmol} / \mathrm{L}$ versus $1.8[1.0,2.4]$, $p=0.021)$ and higher dose of norepinephrine $(0.30[0.15$, $0.50] \mu \mathrm{g} / \mathrm{kg} / \mathrm{min}$ versus $0.15[0.08,0.25] p=0.02)$. At $\mathrm{T} 1$, 81 measurements were available for the index test (NIBRAPG) and for the reference standard (FR-APG). At T2, 76 measurements of the reference standard and the index test were available (it was not measured for 5 patients who had no more norepinephrine infusion at that time). At T3, 71 measurements of the reference standard and the index test were available (it was not measured for 10 patients who had no more norepinephrine infusion at that time). The mean difference of the FR-APG using MAP was $4 \mathrm{mmHg}$ at $\mathrm{T} 1$, ranging from -6 to $28 \mathrm{mmHg}$; also $4 \mathrm{mmHg}$ at $\mathrm{T} 2$ ranging from -22 to $30 \mathrm{mmHg}$ at T2 and $2 \mathrm{mmHg}$ at $\mathrm{T} 3$, ranging from -17 to $16 \mathrm{mmHg}$. The mean difference in the FR-APG using SAP was $10 \mathrm{mmHg}$ at $\mathrm{T} 1$ ranging from -14 to $62 \mathrm{mmHg} ; 8 \mathrm{mmHg}$ at $\mathrm{T} 2$ ranging from -17 to $58 \mathrm{mmHg}$ and $2 \mathrm{mmHg}$ at $\mathrm{T} 3$, ranging from -17 to $16 \mathrm{mmHg}$.

\section{Diagnosis accuracy of the NIBR-APG to detect an FR-APG}

The diagnostic accuracy of the different components of the pressure gradient are detailed in Table 2 and Fig. 2 . Among the six non-invasive approaches using the NIBRAPG to predict a significant FR-APG with either MAP or SAP, the most accurate evaluation was obtained using the average of the three measurements (T1, T2 and T3) of the two upper limbs using NIBP at the brachial artery 


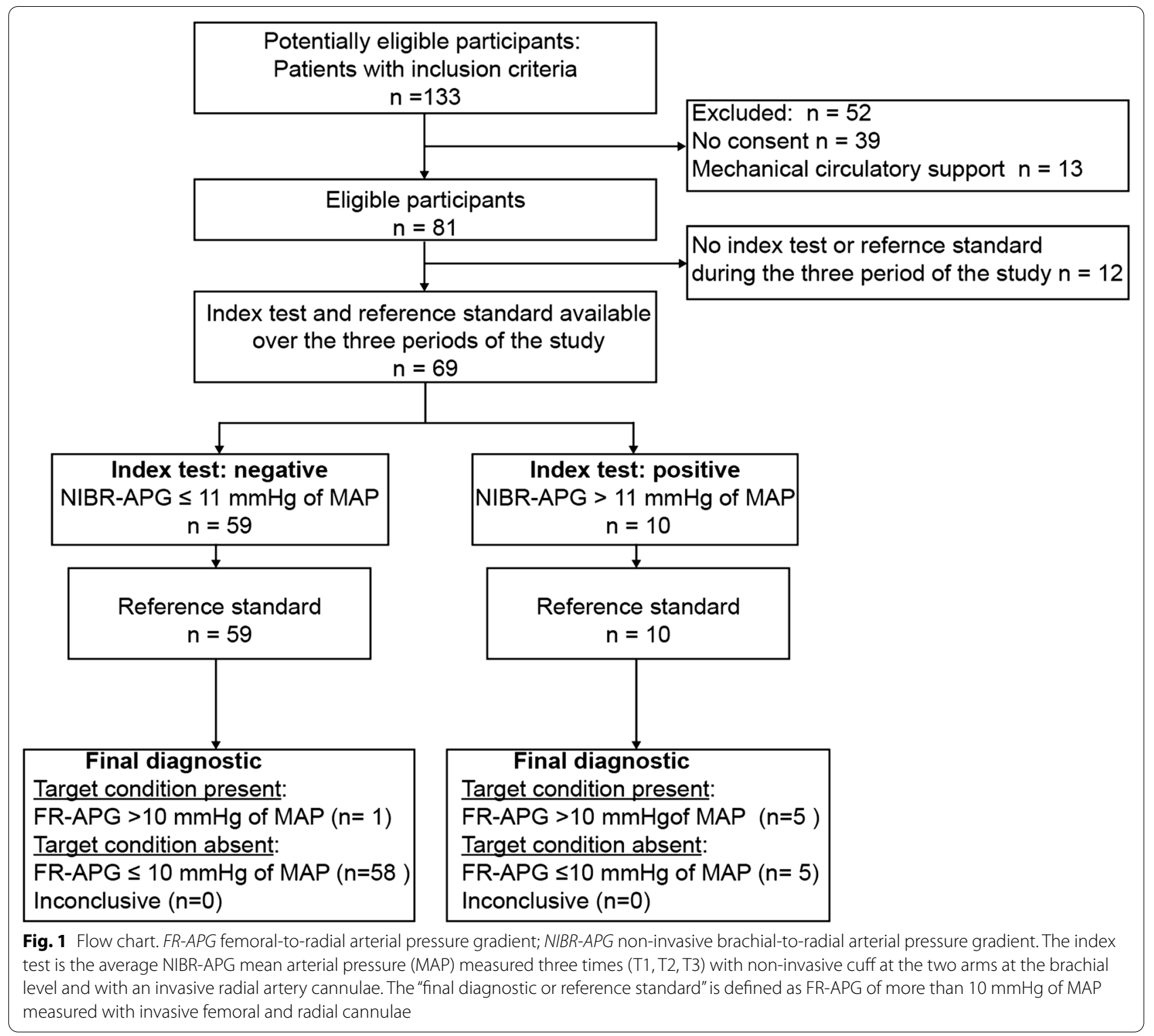

level for the detection a significant FR-APG defined as a MAP difference of more than $10 \mathrm{mmHg}$. The use of a $11 \mathrm{mmHg}$ difference in MAP between the NIBP at the brachial artery and the MAP of the radial artery led to a specificity of $92 \%[67 ; 100]$, a sensitivity of $100 \%$ [95\%CI $83 ; 100$ ] and an AUC ROC of 0.93 [95\%CI 0.81-0.99].

\section{Correlation between NIBR-APG and FR-APG}

When comparing the MAP component of the FR-APG with the MAP of the NIBR-APG, the correlation was $r^{2}=0.34(p<0.001)$ at T1, $r^{2}=0.41 \quad(p<0.001)$ at $\mathrm{T} 2$, $r^{2}=0.21 \quad(p<0.001)$ at T3. When comparing the SAP component, the FR-APG with the SAP of the NIBR-APG, the correlation was $r^{2}=0.36(p<0.001)$ at $\mathrm{T} 1, r^{2}=0.41$ $(p<0.001)$ at T2, $r^{2}=0.35(p<0.001)$ at T3. When averaging the measurements over time (T1 to T3), the SAP component $\left(r^{2}=0.30, p<0.001\right)$ and the MAP component $\left(r^{2}=0.32, p<0.001\right)$ of the FR-APG and the NIBRAPG were significantly correlated (Fig. 3).

\section{Prevalence of the FR-APG}

Overall, at T1, 15 patients, $18.5 \%$ [95\%CI 11-29\%], at T2, 18 patients, $24 \%$ [95\%CI 15-35] and at T3, 8 patients $12.5 \%$ [95\%CI 15-35] had a significant SAP or MAP FRAPG. In terms of SAP and MAP gradient values, at T1, 12 patients (15\%) [95\%CI 8-24] had a significant SAP FR-APG and 10 (12\%) [95\%CI 6-22] had a significant MAP FR-APG. At T2, 10 patients (13\%) [95\%CI 7-23] 
Table 1 Patient's demographic and clinical characteristics at T1

\begin{tabular}{|c|c|c|c|c|}
\hline & All patients & $\begin{array}{l}\text { No significant } \\
\text { FR-APG }\end{array}$ & $\begin{array}{l}\text { Significant } \\
\text { FR-APG }\end{array}$ & $p$ value \\
\hline$n(\%)$ & 81 & $66(81)$ & $15(19)$ & \\
\hline Gender, male/female, $n$ & $53 / 28$ & $44 / 22$ & $9 / 6$ & 0.85 \\
\hline Age, year & $68[58,75]$ & $68[58,75]$ & $70[65,76]$ & 0.365 \\
\hline Height, cm & $168(10)$ & $168(10)$ & $169(11)$ & 0.761 \\
\hline Weight, kg & $80[71,94]$ & $80[71,94]$ & $81[75,87]$ & 0.863 \\
\hline $\mathrm{BMI}, \mathrm{cm} / \mathrm{kg}^{2}$ & $29(5)$ & $29(6)$ & $29(4)$ & 0.848 \\
\hline \multicolumn{5}{|l|}{ Background } \\
\hline Hypertension & $53(66 \%)$ & $41(63 \%)$ & $12(80 \%)$ & 0.344 \\
\hline Diabetes melitus & $23(29 \%)$ & $15(23 \%)$ & $8(53 \%)$ & 0.044 \\
\hline Ischemic cardiomyopathy & $41(51 \%)$ & $36(55 \%)$ & $5(33 \%)$ & 0.231 \\
\hline \multicolumn{5}{|l|}{ Clinical context } \\
\hline Cardiac surgery & 68 & $57(86 \%)$ & $11(73 \%)$ & 0.247 \\
\hline Septic shock & 5 & $4(6 \%)$ & $1(7 \%)$ & 0.904 \\
\hline Other & 8 & $5(8 \%)$ & $3(20 \%)$ & 0.162 \\
\hline \multicolumn{5}{|l|}{ Cardiac and metabolic function } \\
\hline LVEF, \% & $54[40,60]$ & $52[40,60]$ & $56[49,65]$ & 0.144 \\
\hline Lactate, $\mathrm{mmol} / \mathrm{L}$ & $1.95[1.10,2.70]$ & $1.8[1.0,2.4]$ & $2.8[1.6,5.2]$ & 0.021 \\
\hline Temperature, ${ }^{\circ} \mathrm{C}$ & $36.7(0.7)$ & $36.7(0.7)$ & $36.9(1.0)$ & 0.511 \\
\hline $\mathrm{ScvO}_{2}, \%$ & $73[68,77]$ & $73[67,77]$ & $71[70,77]$ & 0.76 \\
\hline Mean NIRS, \% & $69[69,70]$ & $70[69,70]$ & $69[68,69]$ & 0.14 \\
\hline \multicolumn{5}{|l|}{ Fluid } \\
\hline Fluid intake, $\mathrm{mL}$ & $2472[1833,3189]$ & $2475[1805,3275]$ & $2315[2113,2600]$ & 0.731 \\
\hline Fluid loss, mL & $1362[593,2401]$ & $1398[635,2637]$ & $1325[527,1715]$ & 0.507 \\
\hline Fluid balance, $\mathrm{mL}$ & $1320[612,2014]$ & $1300[573,1851]$ & $1775[934,2619]$ & 0.165 \\
\hline Creatinine, $\mu \mathrm{mol} / \mathrm{L}$ & $105[81,138]$ & $103[80,127]$ & $121[89,168]$ & 0.379 \\
\hline \multicolumn{5}{|l|}{ Severity scores } \\
\hline SAPS2 & $35(7)$ & $34(7)$ & $39(10)$ & 0.146 \\
\hline SOFA score & $6[4,7]$ & $5[4,7]$ & $6[5,8]$ & 0.218 \\
\hline \multicolumn{5}{|l|}{ Drugs } \\
\hline Norepinephrine, $\mu \mathrm{g} / \mathrm{kg} / \mathrm{min}$ & $0.16[0.08,0.28]$ & $0.15[0.08,0.25]$ & $0.30[0.15,0.50]$ & 0.02 \\
\hline Epinephrine, $\mu \mathrm{g} / \mathrm{kg} / \mathrm{min}$ & $0.07[0.04,0.10]$ & $0.05[0.04,0.08]$ & $0.09[0.07,0.10]$ & 0.294 \\
\hline Vasopressine, ui/kg/min & $2.40[2.40,2.40]$ & $2.40[2.40,2.40]$ & $2.40[2.40,2.40]$ & 0.378 \\
\hline Dobutamine, $\mu \mathrm{g} / \mathrm{kg} / \mathrm{min}$ & $3.00[2.50,5.00]$ & $2.50[2.50,3.75]$ & $4.00[3.50,4.50]$ & 0.361 \\
\hline Milrinone, $\mu \mathrm{g} / \mathrm{kg} / \mathrm{min}$ & $0.34[0.25,0.47]$ & $0.31[0.25,0.41]$ & $0.40[0.35,0.45]$ & 0.411 \\
\hline \multicolumn{5}{|l|}{ Hemodynamic } \\
\hline $\mathrm{HR}$, beat/min & $78(12)$ & $77(12)$ & $83(11)$ & 0.249 \\
\hline \multicolumn{5}{|l|}{ Radial artery } \\
\hline $\mathrm{SAP}, \mathrm{mmHg}$ & $103(17)$ & $107(16)$ & $89(13)$ & $<0.001$ \\
\hline DAP, mmHg & $54(10)$ & $56(9)$ & $46(10)$ & $<0.001$ \\
\hline $\mathrm{MAP}, \mathrm{mmHg}$ & $69(11)$ & $72(9)$ & $58(10)$ & $<0.001$ \\
\hline \multicolumn{5}{|l|}{ Femoral artery } \\
\hline $\mathrm{SAP}, \mathrm{mmHg}$ & 113(15) & $110(13)$ & $125(18)$ & $<0.001$ \\
\hline DAP, $\mathrm{mmHg}$ & $55(9)$ & $56(8)$ & $50(8)$ & 0.009 \\
\hline MAP, mmHg & $72[68,78]$ & $72[68,78]$ & $70[64,77]$ & 0.315 \\
\hline
\end{tabular}

Variables are expressed as mean (standard deviation) or median [interquartile range] according to their distributions

$B M I$ body mass index; DAP diastolic arterial pressure; $L V E F$ left ventricular ejection fraction; MAP mean arterial pressure; NIRS near-infrared spectroscopy; FR-APG femoral-to-radial arterial pressure gradient; $H R$ heart rate; SAP systolic arterial pressure; SAPS2 Simplified Acute Physiology Score; $S_{C V} \mathrm{O}_{2}$ central venous oxygenation saturation; SIRS systemic inflammatory response syndrome; SOFA Sequential Organ Failure Assessment 
Table 2 Diagnostic accuracy of non-invasive brachial-to-radial (NIBR-APG) arterial pressure gradient to detect a significant invasive mean and systolic femoral-to-radial arterial pressure gradient (FR-APG)

\begin{tabular}{|c|c|c|c|c|c|c|c|c|}
\hline & Approaches & AUC-ROC [95\%Cl] & $\begin{array}{l}\text { Best threshold } \\
(\mathrm{mm} \mathrm{Hg})\end{array}$ & Specificity & Sensitivity & PPV & NPV & Accuracy \\
\hline \multirow[t]{2}{*}{$\begin{array}{l}\text { MAP FR-APG } \\
(\triangle M A P>10 \mathrm{~mm} \\
\mathrm{Hg})\end{array}$} & $\begin{array}{l}\text { NIBR-APG avg } \\
(n=66) \\
(3 \text { measurements })\end{array}$ & 0.93 [0.81-0.99] & $11[3 ; 16]$ & $92[67 ; 100]$ & $100[83 ; 100]$ & $50[21 ; 100]$ & $100[97 ; 100]$ & $91[65 ; 99]$ \\
\hline & $\begin{array}{l}\text { NIBR-APG initial } \\
(n=81) \\
(1 \text { measurement })\end{array}$ & $0.83[0.61-0.95]$ & $5[-6 ; 11]$ & 78 [62-97] & 80 [60-100] & 36 [23-75] & 97 [94-100] & 79 [64-95] \\
\hline \multirow[t]{2}{*}{$\begin{array}{l}\text { SAP FR-APG } \\
(\triangle \mathrm{SAP}>25 \mathrm{~mm} \mathrm{Hg})\end{array}$} & $\begin{array}{l}\text { NIBR-APG avg } \\
(n=66) \\
(3 \text { measurements })\end{array}$ & $0.85[0.71-0.97]$ & $18[-2 ; 27]$ & $84[53 ; 98]$ & $88[63 ; 100]$ & $40[20-83]$ & $97[94.100]$ & $84[53 ; 96]$ \\
\hline & $\begin{array}{l}\text { NIBR-APG initial } \\
(n=81) \\
(1 \text { measurement })\end{array}$ & $0.80[0.61-0.95]$ & $14[3 ; 25]$ & $86[66 ; 97]$ & $75[50 ; 100]$ & $50[28 ; 82]$ & $95[91 ; 98]$ & $86[70 ; 95]$ \\
\hline
\end{tabular}

AUC-ROC area under the curve of the receiver operating characteristic; NPV negative predictive value; PPV positive predictive value; NIBR-APG avg non-invasive brachial to radial arterial pressure gradient averaged from $T 1$ to $T 3$, considering the mean arterial pressure, at the radial level with a catheter and non-invasively on both arms with a cuff at the brachial level; $F R$-APG femoral-radio arterial pressure gradient, considering the mean arterial pressure, at the radial level with a catheter and with a femoral catheter to assess central arterial pressure; NIBR-APG initial non-invasive brachial to radial arterial pressure gradient at T1, considering the mean arterial pressure, at the radial level with a catheter and non-invasively on both arms with a cuff at the brachial level; $M A P$ mean arterial pressure; $S A P$ systolic arterial pressure gradient

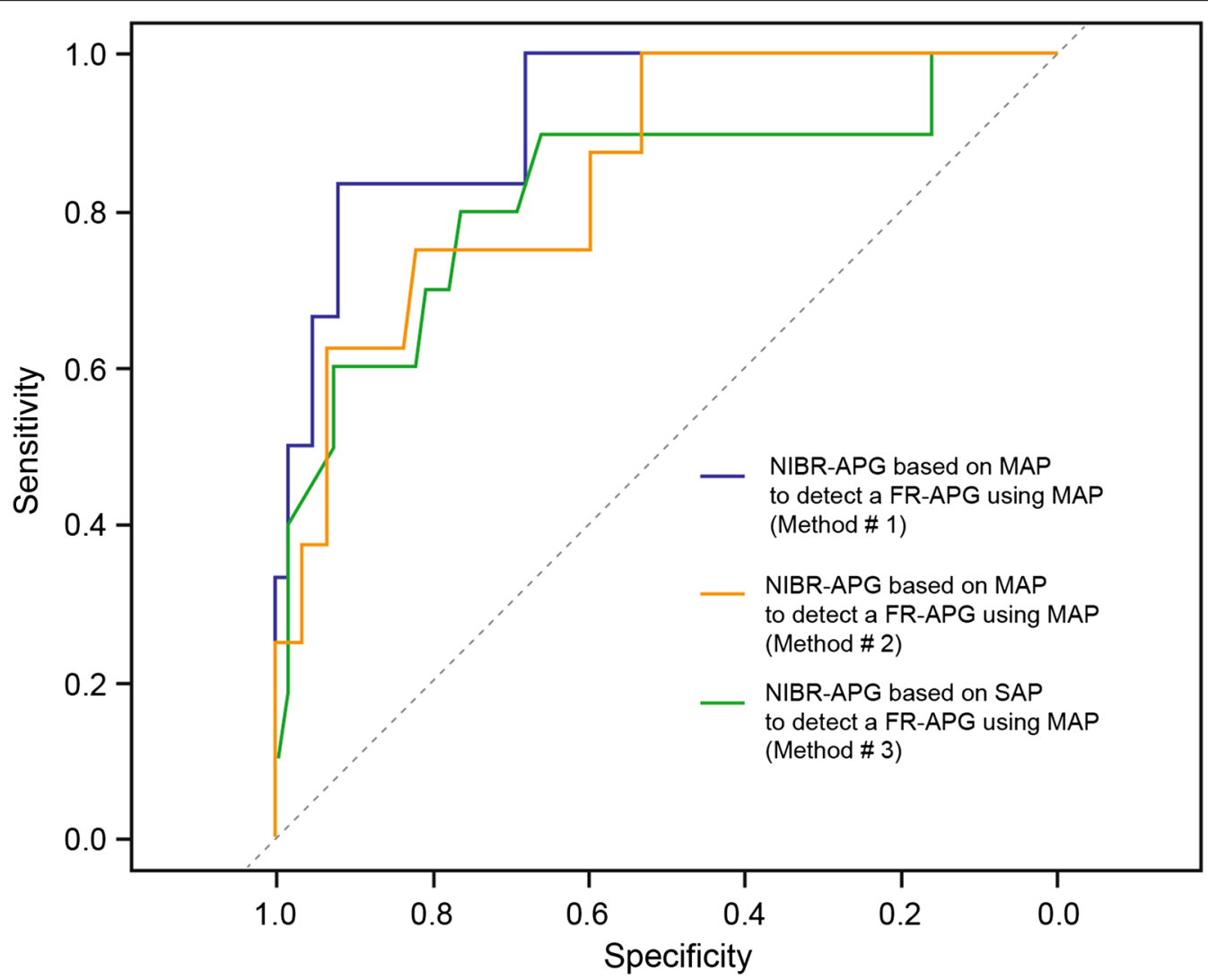

Fig. 2 Receiver operating characteristics curves using three non-invasive methods to estimate the femoral-to-radial arterial pressure gradient (FR-APG) defined in terms of mean arterial pressure (MAP). The first method (purple) is using the average of the three non-invasive measurements (T1, T2 and T3) of the two upper limbs. The second method (orange) is using only non-invasive MAP value at T1. The third method (green) is using only non-invasive systolic arterial pressure (SAP) value at T1. NIBR-APG non-invasive brachial-to-radial arterial pressure gradient; FR-APG is defined as an FR-APG of more than $10 \mathrm{mmHg}$ MAP measured with invasive femoral and radial cannulae 


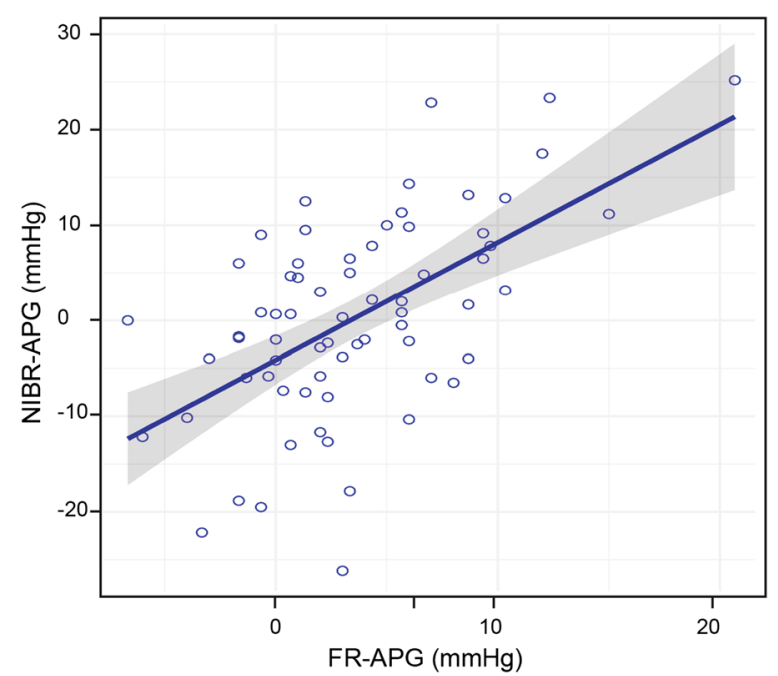

Fig. 3 Scatter plot of the femoral-to-radial arterial pressure gradient (FR-APG) versus non-invasive brachial-to-radial arterial pressure gradient (NIBR-APG). The correlation between the FR-APG in mean arterial pressure (MAP) and the NIBR-APG in MAP was $r^{2}=0.32$ $(p<0.001)$

had both a significant SAP FR-APG and MAP FR-APG. At T3, 7 patients $10 \%$ [95\%CI 7-24] had a significant SAP FR-APG and 3 patients (4\%) [95\%CI 1-12] had a significant MAP FR-APG.

\section{Discussion}

The main finding of our study is that 15 patients among 81 patients $(18.5 \%$ [95\%CI 11-29\%]) had a significant FR-APG in the ICU. Patients with abnormal FR-APG were more frequently diabetes, with higher lactate and received higher doses of norepinephrine. We also found that the average measurements of the two arms every hour, three times, of the NIBR-APG was significantly correlated with the FR-APG. Such measurement can diagnose a significant FR-APG with an AUC ROC of 0.93 [95\%CI 0.81-0.99]. The best threshold was a $11 \mathrm{mmHg}$ difference between the MAP of the brachial NIBP and the MAP of the radial artery pressure, with a specificity of $92 \%[67 ; 100]$ and a sensitivity of $100 \%$ [95\%CI 83; 100].

The prevalence of a significant gradient on our selected group of patients was lower than what has been described during cardiac surgery, but is concordant with another similar ICU study [7]. In Kim study, 37 septic shock patients were monitored with both radial and femoral arterial pressure [7]. Higher FR-APG (using an MAP) $>10 \mathrm{mmHg}$ were observed in $27 \%$ of patients. Femoral pressures were found to be higher than radial particularly in the group receiving high dose of noradrenaline $(>0.1 \mu \mathrm{g} / \mathrm{kg} / \mathrm{min})$ as reported by Dorman et al. [8].
The abnormal FR-APG does not seem to be a rare event in ICU and can certainly affect the therapeutic decision with the risk of excess of fluid [26-31] and vasopressors [32-38] which both have been reported to be harmful.

There is a discrepancy in the different studies in the identification of risk factors of a significant gradient. As previously described in the ICU, significant gradient is associated with higher doses of vasopressors [7, 22, 39, 40]. The association with higher norepinephrine dosage is thought to be part of the physiological mechanism underlying this condition which is consistent with our findings [41, 42]. In fact, vasoactive agents are known to create peripheral vasoconstriction of medium-size arteries $[12,42]$ which may also explain the higher lactate that we observed.

Kanazawa et al. studied different site of measurement along the radial, brachial and the subclavian artery. They found a strong correlation between the distance from the aorta to the site of measurement and the pressure drop from the aorta to the site of measurement [19]. Those findings are consistent with the fact that the noninvasive assessment of the brachial pressure enables the calculation of a gradient with the radial artery that was correlated with the FR-APG. The detection of the MAP gradient seems more accurate than the SAP gradient this could be explained by the reduced accuracy of the oscillometric method to assess SAP compared with MAP [1].

This study has several limitations. First, the association of FR-APG with diabetes has not been reported previously but our number of patients with a significant FRAPG is too small to identify more than one factor using logistic regression. This is, however, the largest reported study with radial and femoral artery measurements in the ICU after Mignini who included 55 patients [13] and the first reporting NIBP to estimate the FR-APG. Second, most of the patients are from a cardiac surgical intensive care unit despite the fact that there were three other medico-surgical ICU involved. This can be explained by the more frequent use of dual radial and femoral artery pressure monitoring in that population. This selection bias could affect the prevalence of the gradient as cardiopulmonary bypass have been described as a significant risk factor for FR-APG $[9,10]$. The precise duration of the FR-APG was not evaluated, but we observed a reduction in the FR-APG over time resulting from reduction in vasoactive agents based on the femoral arterial pressure values. However, the reduction in the severity of the gradient was influenced by an unblinded use of the femoral artery as a target to wean vasoactive agents. In the absence of a femoral artery and detection of an FRAPG, the duration of vasoactive support could be longer. A recent study from our center has shown that the use of combined radial and femoral artery catheter versus radial 
artery alone was associated with a reduction in the duration of vasoactive agents used in the ICU despite using dual radial and femoral arterial pressure in sicker patients [43]. Third, the precision of the NIBP assessment was superior when averaged on three measurements as when only one measurement was made. This is similar to performing more than one thermodilution cardiac output to increase the precision of the measurement. Fourth, there is a risk of biases as the nurse in charge who performed all the measurements was not blind from the reference standard (invasive femoral and radial arterial pressure) when collecting the data of the index test and vice versa. Nevertheless, it is unlikely that the absence of blindness affected the results significantly. Finally, FR-APG can still be present in the absence of vasoactive agents. In our study, we stopped the protocol when those agents were weaned which could led to underestimation of the FRAPG prevalence.

There are numerous advantages of continuous invasive arterial pressure measurement even through the radial artery which include blood gases measurements. If a discrepancy occurs and persist between non-invasive measurements and the radial artery, inserting a femoral arterial line would be suggested. Finally, an indication bias might be present. The use of both radial and femoral arterial pressure monitoring might have been decided for patients at risk of developing such gradients. However, in our institution, the use of both monitoring site is routinely used in up to $70 \%$ of patients in cardiac surgical patients $[9,43]$.

\section{Conclusion}

Significant FR-APG can occur in nearly 1 out of 5 ICU patients. Such can affect significantly the interpretation of the hemodynamic condition of our patient with a risk of fluid or vasopressors overuse. No clear risk factors were linked strongly enough to enable the clinician to rule out the presence of that condition on an epidemiological approach. The NIBP measurement of brachial arterial using a MAP threshold of $11 \mathrm{mmHg}$ can identify those patients and may avoid the use of an invasive femoral catheter. Persistence of a gradient despite modification of therapeutic strategy could warrant more invasive diagnostic strategy. Validation of this hypothesis in a larger cohort of patients should be performed.

\footnotetext{
Abbreviations

AUC: Area under the curve; BP: Blood pressure; Cl: Confidence interval; DAP: Diastolic arterial pressure; FR-APG: Femoral-to-radial arterial pressure gradient; ICU: Intensive care unit; MAP: Mean arterial pressure; NIBP: Non-invasive blood pressure; NIBR-APG: Non-invasive brachial-to-radial arterial pressure gradient; NIRS: Near infrared spectroscopy; ROC: Receiver operating curve; SAP: Systolic arterial pressure gradient; SAPS2: Simplified Acute Physiology Score II; $\mathrm{SCVO}_{2}$ : Central venous oxygen saturation.
}

Acknowledgements

Not applicable.

\section{Authors' contributions}

MJL: study concept and design, acquisition and interpretation of data, drafting of manuscript-statistical analysis, and interpretation of data. DC: acquisition and interpretation of data and critical revision of the manuscript for important intellectual content. JC: acquisition and interpretation of data and critical revision of the manuscript for important intellectual content. KPL: acquisition and interpretation of data and critical revision of the manuscript for important intellectual content. JGG: study concept and design, critical revision of the manuscript for important intellectual content, and interpretation of data. PA: study concept and design, critical revision of the manuscript for important intellectual content, and interpretation of data. YL: study concept and design, critical revision of the manuscript for important intellectual content, and interpretation of data. MA: study concept and design, critical revision of the manuscript for important intellectual content, and interpretation of data. EC: study concept and design, critical revision of the manuscript for important intellectual content, and interpretation of data. LK: study concept and design, critical revision of the manuscript for important intellectual content, and interpretation of data. AD: study concept and design, critical revision of the manuscript for important intellectual content, interpretation of data, and study supervision. All authors are in agreement to be accountable for all aspects of the work, all authors ensured that questions related to the accuracy or integrity of any part of the work are appropriately investigated and resolved. All authors read and approved the final manuscript.

\section{Funding}

Supported by the Montreal Heart Institute Foundation and the Richard I. Kaufman Endowment Fund in Anesthesia and Critical Care. The funding source(s) had no role in the design of the study and collection, analysis, and interpretation of data and in writing the manuscript.

Availability of data and materials

Not applicable.

\section{Declarations}

\section{Ethics approval and consent to participate}

The Local Institutional Review Board for human subjects approved the study protocol (IRB\#: MP-33-2016-1964) and informed consent was obtained before inclusion.

\section{Consent for publication \\ Not applicable.}

\section{Competing interests}

Dr. Denault is speaker and consultant for CAE Healthcare, and speaker for Edwards and Masimo. He received a research grant from Edwards (2019).

\section{Author details}

${ }^{1}$ Centre Hospitalier Louis Pradel, Département d'Anesthésie Réanimation, Hospices Civils de Lyon, 59 Boulevard Pinel, 69500 Bron, France. ${ }^{2}$ Université Claude-Bernard, Lyon 1, Campus Lyon Santé Est, 8 avenue Rockefeller, 69008 Lyon, France. ${ }^{3}$ Carmen Laboratory, IHU OPERA, Inserm U1060, University Claude Bernard Lyon 1, Lyon, France. ${ }^{4}$ Centre de Santé et de Svc, 435 rue Saint Roch, Trois-Rivières, QC G9A 2L9, Canada. ${ }^{5} \mathrm{CHUM}, 1051$ rue Sanguinet, Montreal, QC H2X 3E4, Canada. ${ }^{6}$ Pierre-Le Gardeur Hospital, 911 Montée des Pionniers, Terrebonne, QC J6V 2H2, Canada. ${ }^{7}$ Montreal Heart Institute, Université de Montréal, 5000 rue Belanger, Montreal, QC H1T 1 C8, Canada. ${ }^{8}$ Hôpital du Sacré-Coeur de Montréal, 5400 boul. Gouin O, Montreal, QC H4J 1C5, Canada. ${ }^{9} \mathrm{CHU}$ Amiens-Picardie Site Nord, 2 Place Victor Pauchet, 80080 Amiens, France.

Received: 23 May 2021 Accepted: 14 November 2021

Published online: 27 November 2021 


\section{References}

1. Brzezinski M, Luisetti T, London MJ. Radial artery cannulation: a comprehensive review of recent anatomic and physiologic investigations. Anesth Analg. 2009;109(6):1763-81

2. Kroeker EJ, Wood EH. Comparison of simultaneously recorded central and peripheral arterial pressure pulses during rest, exercise and tilted position in man. Circ Res. 1955:3(6):623-32.

3. Smith EG, Voyles WF, Kirby BS, Markwald RR, Dinenno FA. Ageing and leg postjunctional alpha-adrenergic vasoconstrictor responsiveness in healthy men. J Physiol. 2007;582(Pt 1):63-71.

4. Stern DH, Gerson Jl, Allen FB, Parker FB. Can we trust the direct radial artery pressure immediately following cardiopulmonary bypass? Anesthesiology. 1985;62(5):557-61.

5. Manecke GR Jr, Parimucha M, Stratmann G, Wilson WC, Roth DM, Auge WR, Kerr KM, Jamieson SW, Kapelanski DP, Mitchell MM. Deep hypothermic circulatory arrest and the femoral-to-radial arterial pressure gradient. J Cardiothorac Vasc Anesth. 2004;18(2):175-9.

6. Rich GF, Lubanski RE Jr, McLoughlin TM. Differences between aortic and radial artery pressure associated with cardiopulmonary bypass. Anesthesiology. 1992;77(1):63-6.

7. Kim WY, Jun JH, Huh JW, Hong SB, Lim CM, Koh Y. Radial to femoral arterial blood pressure differences in septic shock patients receiving high-dose norepinephrine therapy. Shock. 2013;40(6):527-31.

8. Dorman T, Breslow MJ, Lipsett PA, Rosenberg JM, Balser JR, Almog Y, Rosenfeld BA. Radial artery pressure monitoring underestimates central arterial pressure during vasopressor therapy in critically ill surgical patients. Crit Care Med. 1998;26(10):1646-9.

9. Fuda G, Denault A, Deschamps A, Bouchard D, Fortier A, Lambert J, Cou ture $P$. Risk factors involved in central-to-radial arterial pressure gradient during cardiac surgery. Anesth Analg. 2016;122(3):624-32.

10. Bouchard-Dechene V, Couture P, Su A, Deschamps A, Lamarche Y, Desjardins G, Levesque S, Denault AY. Risk factors for radial-to-femoral artery pressure gradient in patients undergoing cardiac surgery with cardiopulmonary bypass. J Cardiothorac Vasc Anesth. 2018;32(2):692-8.

11. Pauca AL, Wallenhaupt SL, Kon ND, Tucker WY. Does radial artery pressure accurately reflect aortic pressure? Chest. 1992;102(4):1193-8.

12. Baba T, Goto T, Yoshitake A, Shibata Y. Radial artery diameter decreases with increased femoral to radial arterial pressure gradient during cardiopulmonary bypass. Anesth Analg. 1997;85(2):252-8

13. Mignini MA, Piacentini E, Dubin A. Peripheral arterial blood pressure monitoring adequately tracks central arterial blood pressure in critically ill patients: an observational study. Crit Care. 2006:10(2):R43.

14. Galluccio ST, Chapman MJ, Finnis ME. Femoral-radial arterial pressure gradients in critically ill patients. Crit Care Resusc. 2009:11(1):34-8.

15. Pytte M, Dybwik K, Sexton J, Straume B, Nielsen EW. Oscillometric brachial mean artery pressures are higher than intra-radial mean artery pressures in intensive care unit patients receiving norepinephrine. Acta Anaesthesiol Scand. 2006;50(6):718-21.

16. VanBeck JO, White RD, Abenstein JP, Mullany CJ, Orszulak TA. Comparison of axillary artery or brachial artery pressure with aortic pressure after cardiopulmonary bypass using a long radial artery catheter. J Cardiothorac Vasc Anesth. 1993;7(3):312-5.

17. Gravlee GP, Brauer SD, O'Rourke MF, Avolio AP. A comparison of brachial, femoral, and aortic intra-arterial pressures before and after cardiopulmonary bypass. Anaesth Intensive Care. 1989;17(3):305-11.

18. Rulf EN, Mitchell MM, Prakash O, Rijsterborg H, Cruz E, Deryck YL, Rating W, Schepp RM, Siphanto K, Van der Woerd A. Measurement of arterial pressure after cardiopulmonary bypass with long radial artery catheters. J Cardiothorac Anesth. 1990;4(1):19-24.

19. Kanazawa M, Fukuyama H, Kinefuchi Y, Takiguchi M, Suzuki T. Relationship between aortic-to-radial arterial pressure gradient after cardiopulmonary bypass and changes in arterial elasticity. Anesthesiology. 2003;99(1):48-53.

20. Bossuyt PM, Reitsma JB, Bruns DE, Gatsonis CA, Glasziou PP, Irwig L, Lijmer JG, Moher D, Rennie D, de Vet HC, et al. STARD 2015: an updated list of essential items for reporting diagnostic accuracy studies. BMJ. 2015;351:h5527.

21. Jacquet-Lagreze M, Izaute G, Fellahi JL. Diagnostic accuracy studies: the methodologic approach matters! Anesthesiology. 2017;127(4):728-9.
22. Achino C, Schweizer R, Jacquemet PL, Fellahi JL, Jacquet-Lagreze M. Large underestimation of arterial pressure after vasodilator medication overdose. Br J Anaesth. 2020;125(3):e269-71.

23. Vincent JL, Moreno R, Takala J, Willatts S, De Mendonca A, Bruining H, Reinhart CK, Suter PM, Thijs LG. The SOFA (Sepsis-related Organ Failure Assessment) score to describe organ dysfunction/failure. On behalf of the Working Group on Sepsis-Related Problems of the European Society of Intensive Care Medicine. Intensive Care Med. 1996;22(7):707-10.

24. Le Gall JR, Lemeshow S, Saulnier F. A new Simplified Acute Physiology Score (SAPS II) based on a European/North American multicenter study. JAMA. 1993;270(24):2957-63.

25. Obuchowski NA. Sample size calculations in studies of test accuracy. Stat Methods Med Res. 1998:7(4):371-92.

26. Bouchard J, Soroko SB, Chertow GM, Himmelfarb J, Ikizler TA, Paganini EP, Mehta RL, Program to Improve Care in Acute Renal Disease (PICARD) Study Group. Fluid accumulation, survival and recovery of kidney function in critically ill patients with acute kidney injury. Kidney Int. 2009;76(4):422-7.

27. Boyd JH, Forbes J, Nakada TA, Walley KR, Russell JA. Fluid resuscitation in septic shock: a positive fluid balance and elevated central venous pressure are associated with increased mortality. Crit Care Med. 2011;39(2):259-65.

28. Kasotakis G, Sideris A, Yang Y, de Moya M, Alam H, King DR, Tompkins R, Velmahos $\mathrm{G}$, Inflammation and Host Response to Injury Investigators. Aggressive early crystalloid resuscitation adversely affects outcomes in adult blunt trauma patients: an analysis of the Glue Grant database. J Trauma Acute Care Surg. 2013;74(5):1215-21.

29. Silva JM Jr, de Oliveira AM, Nogueira FA, Vianna PM, Pereira Filho MC, Dias LF, Maia VP, de Neucamp CS, Amendola CP, Carmona MJ, et al. The effect of excess fluid balance on the mortality rate of surgical patients: a multicenter prospective study. Crit Care. 2013;17(6):R288.

30. Woodward CW, Lambert J, Ortiz-Soriano V, Li Y, Ruiz-Conejo M, Bissell BD, Kelly A, Adams P, Yessayan L, Morris PE, et al. Fluid overload associates with major adverse kidney events in critically ill patients with acute kidney injury requiring continuous renal replacement therapy. Crit Care Med. 2019;47(9):e753-60.

31. Miller TE, Mythen M, Shaw AD, Hwang S, Shenoy AV, Bershad M, Hunley C Associations between perioperative fluid management and patient outcomes: a multicentre retrospective study. Br J Anaesth. 2021;126(3):720-9.

32. Latour JG, Leger-Gauthier C. Vasoactive agents and production of thrombosis during intravascular coagulation. 3. Comparative effects of catecholamines. Am J Pathol. 1987:126(3):569-80.

33. Felker GM, Benza RL, Chandler AB, Leimberger JD, Cuffe MS, Califf RM, Gheorghiade M, O'Connor CM, OPTIME-CHF Investigators. Heart failure etiology and response to milrinone in decompensated heart failure: results from the OPTIME-CHF study. J Am Coll Cardiol. 2003;41(6):997-1003.

34. Anantasit N, Boyd JH, Walley KR, Russell JA. Serious adverse events associated with vasopressin and norepinephrine infusion in septic shock. Crit Care Med. 2014;42(8):1812-20.

35. Martin C, Medam S, Antonini F, Alingrin J, Haddam M, Hammad E, Meyssignac B, Vigne C, Zieleskiewicz L, Leone M. Norepinephrine: not too much, too long. Shock. 2015:44(4):305-9.

36. Auchet T, Regnier MA, Girerd N, Levy B. Outcome of patients with septic shock and high-dose vasopressor therapy. Ann Intensive Care. 2017;7(1):43.

37. Daroca-Pérez R, Carrascosa MF. Digital necrosis: a potential risk of highdose norepinephrine. Ther Adv Drug Saf. 2017;8(8):259-61.

38. Hajjar LA, Vincent JL, Barbosa Gomes Galas FR, Rhodes A, Landoni G, Osawa EA, Melo RR, Sundin MR, Grande SM, Gaiotto FA, et al. Vasopressin versus norepinephrine in patients with vasoplegic shock after cardiac surgery: the VANCS randomized controlled trial. Anesthesiology. 2017;126(1):85-93.

39. Nakayama R, Goto T, Kukita I, Sakata R. Sustained effects of plasma norepinephrine levels on femoral-radial pressure gradient after cardiopulmonary bypass. J Anesth. 1993:7(1):8-16.

40. De Hert SG, Vermeyen KM, Moens MM, Hoffmann VL, Bataillie KJ. Centralto-peripheral arterial pressure gradient during cardiopulmonary bypass: relation to pre- and intra-operative data and effects of vasoactive agents. Acta Anaesthesiol Scand. 1994;38(5):479-85 
41. Currigan DA, Hughes RJ, Wright CE, Angus JA, Soeding PF. Vasoconstrictor responses to vasopressor agents in human pulmonary and radial arteries: an in vitro study. Anesthesiology. 2014;121(5):930-6.

42. Goto K, Saito SY, Ishikawa T. Enhanced vasoconstriction to alpha1-adrenoceptor stimulation during cooling in mouse cutaneous plantar arteries. Eur J Pharmacol. 2014;742:1-7

43. Bouchard-Dechene $\mathrm{V}$, Kontar L, Couture P, Pérusse $\mathrm{P}$, Levesque $\mathrm{S}$, Lamarche Y, Denault AY, the Montreal Heart Institute Research Team.
Radial-to-femoral pressure gradient quantification in cardiac surgery. J Thorac Cardiovasc Surg. 2021. https://doi.org/10.1016/j.xjon.2021.07.031.

\section{Publisher's Note}

Springer Nature remains neutral with regard to jurisdictional claims in published maps and institutional affiliations.
Ready to submit your research? Choose BMC and benefit from:

- fast, convenient online submission

- thorough peer review by experienced researchers in your field

- rapid publication on acceptance

- support for research data, including large and complex data types

- gold Open Access which fosters wider collaboration and increased citations

- maximum visibility for your research: over 100M website views per year

At BMC, research is always in progress.

Learn more biomedcentral.com/submissions 